\title{
Neuropathology of HAND With Suppressive Antiretroviral Therapy: Encephalitis and Neurodegeneration Reconsidered
}

\author{
Benjamin B. Gelman ${ }^{1}$ \\ Published online: 10 April 2015 \\ (C) The Author(s) 2015. This article is published with open access at Springerlink.com
}

\begin{abstract}
HIV-1 infiltrates the central nervous system (CNS) during the initial infection and thereafter plays a persistent role in producing CNS dysfunction as the disease progresses. HIVassociated neurocognitive disorders (HAND) are highly prevalent in HIV-infected patient populations, including currently infected patients with good access to suppressive antiretroviral therapy (cART). cART decreased the severity of CNS dysfunction dramatically and, in doing so, upended the neuropathological foundation of HAND pathophysiology. It is clear that the working concept of pathophysiology prior to cART, which was driven by inflammation, encephalitis, and neurodegeneration, needs to be replaced. The NeuroAIDS field is reluctant to take that important step. This review explores the fact that the neuropathological concept that drove the field before the era of cART no longer seems to fit with what is commonly observed in patients treated successfully with cART. The field clings to the pre-cART idea that HAND is sequentially driven by virus replication in CNS, brain inflammation (encephalitis), and neurodegeneration. Neurovirological, clinicopathological, and gene expression correlations in cARTtreated patients, however, provide little strong support for it. Introducing cART into clinical practice decreased HIVE,
\end{abstract}

This article is part of the Topical Collection on Central Nervous System and Cognition

Benjamin B. Gelman

bgelman@utmb.edu

1 Departments of Pathology and Neuroscience \& Cell Biology, University of Texas Medical Branch, 301 University Blvd., Keiller 3.118A, Route 0609, Galveston, TX 77555-0609, USA inflammation, and degeneration but did not cure HAND. Brain gene array data suggest that the neurovascular unit is a critical target in virally suppressed patients with HAND. The NeuroAIDS field needs an infusion of new ideas to steer research toward issues of the highest relevance to virally suppressed patients. With no suitable replacement immediately within reach, devaluating formative ideas is understandably difficult to accept. The cliniconeuropathological correlation in virally suppressed patients needs to be better defined.

Keywords Antiretroviral therapy · Dementia - Encephalitis · HIV/AIDS · HIV-associated neurocognitive disorders ·

Metabolic encephalopathy $\cdot$ Neurodegeneration .

Neurovirology

\section{Introduction}

Central nervous system (CNS) infection is established early in the course of HIV/AIDS. CNS pathology was a highly prevalent feature in the natural history of patients who were not virally suppressed. The topic of HIV infection of the CNS has enjoyed a sustained period of scientific and clinical interest for over 30 years. The neuropathology of end-stage AIDS has been illustrated comprehensively and is well-described in many excellent book chapters and review articles [1, 2]. Images of the histopathology can be easily acquired in cyberspace in these times and there seems little need to illustrate them yet again. Updating a fast-moving field is potentially useful nevertheless to discuss the existing body of knowledge with a changed perspective. Current times have not been propitious for the discovery of exciting new developments in the neuropathology of HIV/AIDS. The paucity of recent articles with high impact makes an update of neuropathology 
challenging, but also heightens opportunities for analysis, reflection, and commentary. The need for new and genuinely novel ideas in this field has never been greater. HIV-infected patients and the new corps of enterprising young practitioners both deserve to inherit a scientific legacy that points in a clear direction that focuses on relevant problems. Accordingly, this article provides commentary with the aim of spurring new investigators to propel the field creatively forward.

There is a virtual haze of comorbid changes and/or agerelated pathologies that can occur in HIV-infected subjects. When one wipes away the haze in patients who are compliant with taking suppressive antiretroviral therapy (cART), the overwhelmingly dominant HAND-associated neuropathology observed at autopsy is "no specific neuropathology." The lack of a specific neuropathological change, which became the most common outcome at autopsy at the close of the twentieth century, has led to a dearth of novel breakthroughs in the neuropathological literature for at least 15 years. The medical literature favors positive data and shuns negativity. Composing a scholarly treatise regarding the disappearance of pathologies that were prevalent formerly is perhaps more challenging than describing the panoply of CNS changes observed before the era of cART. Present circumstances in the era of cART have benefited our patients substantially, but we still have not canonized a new cliniconeuropathological correlation for HIVassociated neurocognitive disorders (HAND). With no clearcut replacement for the "old" neuropathology to guide us in the era of cART, one can reasonably wonder what the actual mechanism of HAND really is. Therapeutic strategies of current interest are based primarily upon neuropathologies that do not occur with high prevalence. Given the low prevalence of those pathologies in virally suppressed patients, their clinical relevancy to HAND, which has an estimated prevalence of up to $50 \%$ [3], becomes increasingly dubious.

From a historical perspective, the most important cliniconeuropathological concept in the field was established prior to the era of cART in patients in whom virus replication was not suppressed medically. In those patients, progression to end-stage AIDS was virtually certain to occur and about $20 \%$ of patients had HIV encephalitis (HIVE) at autopsy [4, 5]. This neuropathological lesion in the brain was first labeled a "subacute" type of inflammatory process characterized by several features in combination, the most important of which was evidence of HIV replication in mononuclear phagocytes (macrophages and microglial cells).

\section{Microglial Nodules}

Foremost among the histological changes in HIVE was the presence of microglial nodules (MGN), which are activated microglial cells arranged in clusters of variable sizes. Microglial cells are the resident histiocytes of the CNS and, combined with brain macrophages, are the major reservoir of replicating HIV in the brain. Unlike macrophages which originate in bone marrow, microglial cells arrive early in development from the yolk sac and can reproduce locally in the CNS [6]. MGNs can occur in either gray or white matter almost anywhere in the CNS. They can be striking in their abundance in the tissue but are not diagnostic for HIVE because they may be observed in several other kinds of brain infection. When the MGNs are immunostained for HIV proteins such as gp41, HIVp24, or gp120 they are considered to be specific to HIV infection of these cells. Over the years, some confusion was produced because case material often contained an abundance of MGNs without other lesions associated with HIVE (to be discussed). These cases still may be diagnosed "microglial nodule encephalitis" (MGNE) in lieu of HIVE because other infectious agents could not be readily ruled out. It was a critical diagnostic dilemma prior the cART era because encephalitis caused by opportunistic pathogens such as cytomegalovirus and Toxoplasma ghondii also produced MGNs in immunosuppressed patients with AIDS [1, 2, 7••]. The neurovirological underpinnings of HIVE and MGNE diagnoses were recently unraveled. It was shown that HAND with HIVE and HAND with MGNE both are associated with high rates of HIV replication (HIV RNA) in the brain. As expected, brain HIV replication is much higher in HIVE than MGNE, which suggests that there is a neuropathological progression of the disease from lower to higher replication rates. In patients with HAND without either of the two diagnoses, brain HIV RNA and DNA were not significantly higher than patients without HAND. Interestingly, HAND without these two pathologies was significantly correlated with HIV replication in blood plasma, but not in the brain or CSF. Apparently, systemic viral suppression is just as or more critical than viral suppression within the CNS in these patients $[7 \bullet \bullet]$.

\section{Multinucleated Giant Cells and Macrophages in HIVE}

The "hallmark" lesion of HIVE is the multinucleated giant cell (MNGC) [5]. These cells represent mononuclear phagocytes productively infected with HIV that have undergone cellular fusion mediated by the HIV fusion protein gp41. MNGCs are most often observed around blood vessels and resemble other kinds of macrophage giant cells in their general appearance such as those seen in granulomatous inflammation. They are readily distinguishable from other kinds of macrophage giant cells by their positive immunostaining with antibody against HIV proteins, the lack of associated granulomatous inflammation, and the clinical and pathological context in which they are observed. This feature alone is generally adequate to make the diagnosis of HIVE in an infected patient; other features of HIVE described herein are almost always 
present as well. Many single macrophages and/or clusters of macrophages are usually present in HIVE. They are most often seen around the blood vessels in white matter and often contain hemosiderin pigment. Macrophages productively infected with HIV-1 in the brain generally have an M2 ("repair") phenotype; they are CD163+, CD14+/CD16+, and they contain stainable HIV-1 antigens $[8,9]$.

\section{Other Inflammatory Cell Changes in HIVE}

Ancillary and often nonspecific neuropathological changes beyond the key features mentioned above can contribute to a wide spectrum of changes in HIVE. In isolation, these changes are seldom specific enough to diagnose HIVE, but when observed in combination with other features they contribute to a highly characteristic constellation of changes. Diffuse microglial cell activation ("diffuse microgliosis") is commonly observed in HIV-infected brain tissue. It refers to an increase in the number of cells with a dark-stained rod-shaped nucleus scattered in the brain tissue. An increase in the density of "rod cells," which are the elongated hematoxylin-stained nuclei, can occur with or without the formation of MGNs. They are usually present in HIVE and MGNE, but frequently they are observed without either of the two diagnoses. The expansion and heightened ramification of the cytoplasmic processes of the activated microglial cells are not visible in routinely stained tissue sections but are recognized with immunostaining for HLA-D, Iba-1, ferritin, and other antigens $[10,11]$. Other inflammatory cells that can be observed in HIVE include scattered perineuronal lymphocytes. CNS T lymphocytes are not required to make the diagnosis of HIVE and they lack specificity when observed. Indeed, their presence in high abundance serves to heighten suspicion that another agent that can cause encephalitis might be present. Even though lymphocytic infiltration is seldom the most telling or prominent, brain lymphocyte subsets conduct regular immune surveillance in the CNS and still could play a seminal role in the pathophysiology of HIVE such as with neuronal killing [12]. Infiltration of the brain with acute inflammatory cells such as neutrophils and eosinophils in HIVE is not likely to be important because they do not host HIV infection. The basic nature of the inflammatory reaction to HIV infection in the CNS is generally considered to be "subacute" given the lack of neutrophils.

\section{Astrocyte Changes in HIVE}

In HIVE astrocyte hypertrophy and glial scarring are observed best in brain white matter. The hypertrophy is a nonspecific reaction that occurs in all types of brain injury. HIV-infected astrocytes are not morphologically different in a routine neuropathological examination. Diagnostically, their chief value is in establishing that damage has occurred in the setting of inflamed brain tissue. The role of astrocytes remains of great interest because in vivo and in vitro data indicate that astrocytes can be infected with HIV [13]. They also can participate in antigen presentation [14]. HIV-infected astrocytes do not produce assembled viruses very effectively and are not likely to be the main contributor to CNS HIV replication rates. Astrocyte infection is very important because of its potential ability to support viral latency in the brain. In cART-treated and naïve patients both, reverse transcribed HIV DNA is integrated into the host cell genome and becomes "latent" in a highly limited number of host cells. Virally latency is defined as HIV DNA integrated into the host cell genome that is transcriptionally silenced and immunologically inert, yet capable of transcriptional reactivation to produce assembled viruses that are fit to infect other host cells. Upon stopping cART, it is the latent pool of HIV DNA that "reseeds" active HIV replication and prevents the complete eradication of HIV infection. Data thus far indicate that integrated HIV DNA is present in a substantial number of astrocytes in patients with HIVE [15••]; it remains unclear whether the astrocyte pool of HIV DNA contributes to the pool of latent HIV that reseeds the CNS with replicating HIV when cART is stopped. If so, the astrocyte reservoir of latent HIV is a highly significant pool of virus that would need to be dealt with in therapies to eradicate the virus from the CNS.

\section{Neuronal Changes and "Neurodegeneration" in HIVE}

In the era of cART, the literature on neuronal death and dropout in HIV-infected people remains highly difficult to judge in terms of its clinical relevancy. The difficulties are quite complex and have been discussed [1]. The essence is that with HIVE, inflammation damages neuronal viability and leads some of the cells to drop out. In patients with HIVE, it can be logically argued that neuronal dropout is contributory to HAND, and that HIV-associated dementia is a type of neurodegenerative disease. Pursuing a "neuroprotective" strategy in model systems that mimic HIVE is of clinical relevance in patients with HIVE. The field continues to hold that, when HIVE is not present, inflammation drives HAND and fuels neurodegeneration. But the same underlying neuropathology in virally suppressed patients without HIVE has never been clearly substantiated [16]. That in turn begs the question of why protecting against neurodegeneration (in parallel with reducing inflammation and viral replication) remains a mainstay in the search for pharmacotherapies for HAND.

If one broadens the definition of neurodegeneration to include nonlethal and/or reversible changes in neuronal function, then neuroprotection of virally suppressed individuals 
remains a logical therapeutic approach. Evidence which supports this concept includes brain images showing functional adaptation occurring in HIV-infected patients who do not have neurocognitive impairment $[17,18,19 \bullet]$. Neurochemical data from autopsy specimens also reveal that nonlethal synaptic accommodation of neurotransmitter systems is related to HAND, including heightened neocortical dopaminergic tone [20•]. Adopting a broader functional definition of neurodegeneration is, however, at odds with the traditional definition of neurodegenerative diseases such as Alzheimer's or Parkinson's diseases. Those diseases are defined classically as being due to an irreversible neuronal death, cell dropout, and clear-cut morphological destruction of neuronal elements; their clinical manifestations only occur after a very large proportion of the neuronal elements have been destroyed. Those kinds of neuropathological changes have not been observed in recent times with consistency in patients with HAND, whereas nonlethal changes in neuronal function probably do occur $[20 \bullet, 21 \bullet \cdot]$. These milder types of changes are likely to underlie the milder neurocognitive impairments that are observed. Abnormal neuronal function (versus death and dropout) is akin to the metabolic types of encephalopathies, which are traditionally described in a completely different chapter of our neuropathology textbooks. Thus, neuroprotection from metabolic types of damage in patients with viral suppression seems an apt description of what is probably happening (versus neuronal death and dropout).

With survival to older ages in virally suppressed patients, roles of biological and pathological brain aging (such as Alzheimer's or Parkinson's diseases) have been suggested to be potentially interactive with HAND at the histomorphological level. The underlying hypothesis is that the intersection of ageassociated and HIV-associated neurodegeneration could mutually exacerbate each other. The neuropathological literature on this is fragmented, controversial, and inconclusive [22•]. As noted, the case for neuronal death, dropout, and classical neurodegeneration in virally suppressed patients with HAND is increasingly difficult to substantiate. That makes potential synergy with age-related neurodegenerative diseases at the mechanistic level difficult to accept. It is true that neuropathological elements of classical neurodegenerative diseases have begun to appear in older people with HIV infection. And surveys show that elderly people with HIV infection are more vulnerable to neurocognitive impairment. But such changes also begin to occur in aging populations that are not HIV infected. One can just as logically hypothesize that age- and HIV-associated effects could run in parallel with no mechanistic overlapping, and contribute additively to neurocognitive declines in more elderly HIV-infected people. The postulated evolution of a neuropathologically distinct subtype of HAND in people who have age-associated neuropathologies remains to be convincingly validated. It remains unlikely to be disproved completely in the near future, in part because proving a negative proposition is difficult to do and also because negative data can be seen as unwelcome news.

\section{White Matter Changes in HIVE}

White matter damage adds to the spectrum of changes in HIVE. The white matter changes can vary in type, intensity, and their distribution in the brain. White matter edema leads to loss of staining of the myelin ("pallor"). Outright acute destruction of myelin due to exuberant HIV-1 replication and inflammation can occur rarely in HIVE, but more typically slow myelin loss and its phagocytosis by macrophages are restricted to perivascular sectors [23, 24]. Brain imaging studies suggest that focal white matter anomalies are relatively common in HIV-infected patients, including many virally suppressed patients who do not have HIVE, but the neuropsychological implications of that still have not been elucidated clearly [25].

\section{The Neurovascular Unit and Its Potential Importance in the Cliniconeuropathological Correlation}

Gaps in our understanding of the cliniconeuropathological correlation of HAND persist. Each of the neuropathological features reviewed above in the context of their clinical relevancy (or lack of) has been discussed recently [1]. Recent experience has shown convincingly that the overwhelming majority of patients today who are virally suppressed do not have HIVE or MGNE. As well, most of these patients do not have substantial HIV replication in brain tissue [7••] and they do not have an inflammatory gene expression profile in brain tissue $[21 \bullet \cdot$. Of all the CNS structures that are abnormal in virally suppressed patients with HAND, the neurovascular unit (NVU) may be the most important regarding the cliniconeuropathological correlation. The NVU refers to the blood vessel compartment in the CNS and its interface with a web of physical and functional interactions with brain parenchyma [26-28]. The interconnected parts include astrocyte foot processes, neuronal processes and synapses, endothelial cells, pericytes, perivascular macrophages and microglial cells, CNS extracellular spaces, the subarachnoid spaces and cerebrospinal fluid, and perhaps most importantly, blood plasma. Subtle changes in blood plasma are prevalent in HIVinfected patients [29-31], and systemic changes as reflected in the vascular compartment are indeed significantly correlated with HAND. The anemia of chronic inflammation (low red cell volume), and increased plasma lipopolysaccharide due translocation of gut bacteria are both significantly correlated with HAND [32•, 33]. As noted above, the neurovirological correlation in virally suppressed patients with HAND may be weaker than the correlation with plasma viral replication $[7 \bullet \bullet]$. 
The NVU is, therefore, situated anatomically at the critical junction between CNS parenchyma and the plasma compartment at the luminal surface of vascular endothelial cells. Its best-known function is maintaining a highly selective physical barrier between blood plasma and brain parenchyma known as the blood-brain barrier (BBB). In HIVE, the BBB is leaky and leads to cerebral edema by permitting blood plasma to enter the CNS. Experimental systems suggest that this could be detrimental to brain function. Cerebral edema and opening of the $\mathrm{BBB}$ is, however, a nonspecific response to CNS injury. It is not clear whether it reflects an epiphenomenon of brain inflammation versus a substantial cause of brain dysfunction in HIVE. As well, we do not know whether it occurs substantially in HAND patients taking cART who do not have HIVE, encephalitis, or other ongoing neuropathological process. Because the BBB is damaged in HIVE, which in turn is correlated with HAND, it is not surprising that the BBB disturbance was correlated with HAND in studies before the era of cART [23].

Beyond acting as a physical barrier, other functions of the NVU could be perturbed in cART-treated patients who are virally suppressed. As noted, endothelial cell surfaces in contact with blood plasma transmit signals to neurons and glia via the NVU (without apparent breakdown in the BBB). These processes may still be perturbed in virally suppressed patients who have low level inflammatory changes in blood plasma [28-31, 32, $33-35]$. Another key role of the NVU is the regulation of microvascular blood flow. The inhibitory neurotransmitter gamma aminobutyric acid (GABA) controls changes in regional microvascular blood flow via the NVU through influences on contractility of pericytes around capillaries and arteriolar smooth muscle [26-28]. Studies using blood oxygenation level-dependent (BOLD) functional magnetic resonance imaging (fMRI) show convincingly that blood flow in specific brain regions is perturbed in cART-treated patients, with and without HAND [19•]. GABAergic transmission is well-known to be abnormally low in HIV-infected brain tissue $[21 \bullet \bullet$. Thus, abnormal GABAergic neural transmission might be involved with the changes in microvascular blood flow in virally suppressed patients.

Compelling evidence that the NVU is probably important in HAND without HIVE was obtained from a recently reported brain gene expression profile [21••]. The study examined over 56,000 gene transcripts in neuropsychologically impaired people with HIVE who lacked CNS viral suppression, and also in people who were virally suppressed in the blood and brain and did not have HIVE. Three different sectors of the brain were examined. Over 1000 brain transcripts were regulated in HAND with HIVE. In sharp contrast, brain gene regulation was highly restricted in virally suppressed patients with HAND without HIVE. These individuals had abnormal transcription regulation limited to a network of brain genes expressed by endothelial cells. Many of these endothelial-type molecules are shed; their concentrations are increased in blood plasma and are not completely restored to normal in virally suppressed patients [35]. The implication overall is that perturbation of neurovascular biology may be a keystone process in patients who are virally suppressed, whereas in the patients without viral suppression, brain inflammation is a more prevalent force. Heightened awareness that there are two types of HAND with divergent neurovirological $[7 \bullet \bullet]$ and brain transcriptional $[21 \bullet \bullet]$ patterns was suggested two decades ago before the introduction of effective viral suppression [36].

\section{Neuropathology and Eradicating Latent HIV in the CNS}

In 2015, the HIV/AIDS research enterprise worldwide is engaged in an effort to cure AIDS. The effort is focused on eradicating from the body a small pool of genomically integrated but transcriptionally silent cellular HIV DNA. This "latent" pool of HIV DNA is apparently not diminished using current cART formulations; when cART is stopped, the latent HIV pool "reseeds" the pool of replicating virus in the body [37]. The nature of the latent pool of HIV DNA in the CNS has not been characterized as yet, and some have questioned whether its eradication from the body is scientifically feasible. From neurovirological data obtained at autopsy, it is well documented that genomic HIV DNA is present in many brain specimens. In many patients, there is substantial divergence between the size of the HIV DNA pool and the replicating pool (HIV RNA) [7••]. It is evident that the CNS compartment can be variously enriched with, or depleted of, HIV DNA relative to the viral replication rate. Since cells that host latent HIV are likely to be immunologically "inert" and invulnerable to immune surveillance [37], there is very little reason to believe that pathological inflammation such as that observed in HIVE would be linked to it. Similarly, "neurodegeneration" driven by CNS inflammation would be unlikely to be associated with the CNS pool of latent HIV DNA. A recent report suggested that a transcriptionally silenced HIV DNA pool in 10 infected brain specimens was related to high expression of the microglial cell transcription factor BCL11b [38]. It awaits independent replication in more patients, yet intriguingly suggests that repressing the pool of latent HIV DNA in microglial cells is potentially feasible.

Whether HAND is associated with enrichment of the CNS pool of latent HIV DNA (relative to the CNS replication rate) is not clear. Current thinking on this topic is speculative at best. Available data on HIV RNA and total HIV DNA from autopsy brain specimens show that a very low viral replication rate (and low total HIV DNA concentration) in the CNS, as seen in virally suppressed patients, is not substantially correlated with HAND. In contrast, very high rates of viral replication in the CNS (with high concentrations of HIV DNA), as seen in HIVE patients not taking cART, are correlated significantly 
with HAND [7••]. Inclusion of the latter type of patients in most surveys is what drives the significance of this correlation overall. In a follow-up to that study, the authors measured integrated HIV DNA (a more specific measure of viral latency in tissue) using the two-step Alu-gag assay in 29 of the brain specimens. The neurovirological correlation using integrated HIV DNA (both the coefficient and the regression line) was not different from results using total HIV DNA or HIV RNA. The result favors the hypothesis that the latent pool of HIV in the brain per se is no more influential to the cliniconeurovirological correlation than are the other two viral pools that were measured (the author's unpublished observation).

\section{Conclusions: the Future}

The neuropathology of HIV infection changed drastically when cART was introduced into clinical practice. The neuroAIDS field clings to most tenets of the "old" pathology of unsuppressed patients, especially the concepts that encephalitis, neurodegeneration, and CNS HIV replication are the key drivers of HAND. Once considered seminal to the field, those tenets are of increasingly lower relevance clinically. The lack of specific pathoanatomical changes in virally suppressed patients means that the cliniconeuropathological correlation of HAND is driven by subtler and potentially reversible neurometabolic changes, such as those associated with synaptic transmission and neural plasticity [20•]. Correlation between HAND and systemic pathologies also implies a possible metabolic encephalopathy that is one component of a systemically driven illness. Establishing a new conceptual framework is painful and risks "throwing the baby out with the bath water." But the NeuroAIDS field is ripe for a change. The next generation of practitioners and scientists faces a very stiff headwind to meet that challenge.

\section{Compliance with Ethics Guidelines}

Conflict of Interest Benjamin B. Gelman declares no conflict of interest.

Human and Animal Rights and Informed Consent This article does not contain any new studies with human or animal subjects performed by the author.

Open Access This article is distributed under the terms of the Creative Commons Attribution 4.0 International License (http:// creativecommons.org/licenses/by/4.0/), which permits unrestricted use, distribution, and reproduction in any medium, provided you give appropriate credit to the original author(s) and the source, provide a link to the Creative Commons license, and indicate if changes were made.

\section{References}

Papers of particular interest, published recently, have been highlighted as:

- Of importance

-• Of major importance

1. Gelman BB and Moore DJ. HIV-1 neuropathology. Chapter 7.4. In: Gendelman HE, Grant I, Everall IP, Fox HS, Gelbard HA, Lipton SA, and Swindells S, editors. The Neurology of AIDS, 3rd ed. 2011. 978-0-19-539934-9011.

2. Petito CK. Neuropathology of acquired immunodeficiency syndrome. In: Nelson JS, Parisi JE, Schochet Jr SS, editors. Principles and practice of neuropathology. Saint Louis: Mosby; 1993. p. $88-108$.

3. Heaton RK, Clifford DB, Franklin DR, et al. HIV-associated neurocognitive disorders persist in the era of potent antiretroviral therapy: CHARTER Study. Neurology. 2010;75(23):2087-96. doi: 10.1212/WNL.0b013e318200d727.

4. Budka H, Wiley CA, Kleihues P, et al. HIV-associated disease of the nervous system: review of nomenclature and proposal for neuropathology-based terminology. Brain Pathol. 1991;1(3):14352. doi:10.1111/j.1750-3639.1991.tb00653.x.

5. Budka H. Multinucleated giant cells in brain: a hallmark of the acquired immune deficiency syndrome (AIDS). Acta Neuropathol. 1986;69(3-4):253-8. doi:10.1007/BF00688301.

6. Ginhoux F, Lim S, Hoeffel G, Low D, Huber T. Origin and differentiation of microglia. Front Cell Neurosci. 2013;7:45. doi:10. 3389/fncel.2013.00045.

7.• Gelman BB, Lisinicchia JG, Morgello S, et al. Neurovirological correlation with HIV-associated neurocognitive disorders and encephalitis in a HAART-era cohort. J Acquir Immune Defic Syndr. 2013;62(5):487-95. doi:10.1097/QAI.0b013e31827flbdb. This is the only neurovirological correlation done in clinically documented HAND patients with and without HIV encephalitis. A large consortium shows that brain HIV replication is a key driver of HAND in patients not virally suppressed with encephalitis, but brain HIV replication is not significantly higher in virally suppressed HAND patients without encephalitis. This lends added support for the concept of splitting HAND into two neuropathological types as suggested in reference 20 .

8. Fischer-Smith T, Croul S, Sverstiuk AE, et al. CNS invasion by CD14+/CD16+ peripheral blood-derived monocytes in HIV dementia: perivascular accumulation and reservoir of HIV infection. J Neurovirol. 2001;7(6):528-41.

9. Roberts E, Masliah E, Fox HS. CD163 identifies a unique population of ramified microglia in HIV encephalitis (HIVE). J Neuropathol Exp Neurol. 2004;63(12):1255-64.

10. Gelman BB. Diffuse microgliosis associated with cerebral atrophy in the acquired immunodeficiency syndrome. Ann Neurol. 1993;34(1):65-70. doi:10.1002/ana.410340112.

11. Xing HQ, Hayakawa H, Gelpi E, et al. Reduced expression of excitatory amino acid transporter 2 and diffuse microglial activation in the cerebral cortex in AIDS cases with or without HIV encephalitis. J Neuropathol Exp Neurol. 2009;68:99-209. doi:10.1097/ NEN.0b013e31819715df.

12. Petito CK, Torres-Muñoz JE, Zielger F, McCarthy M. Brain CD8+ and cytotoxic $\mathrm{T}$ lymphocytes are associated with, and may be specific for, human immunodeficiency virus type 1 encephalitis in patients with acquired immunodeficiency syndrome. J NeuroVirol. 2006;12(4):272-83.

13. Gorry PR, Ong C, Thorpe J, Bannwarth S, Thompson KA, et al. Astrocyte infection by HIV-1: mechanisms of restricted virus 
replication, and role in the pathogenesis of HIV-1-associated dementia. Curr HIV Res. 2003;1:463-73. doi:10.2174/ 1570162033485122.

14. Dong Y, Benveniste EN. Immune function of astrocytes. Glia. 2001;36(2):180-90.

15.• Churchill MJ, Gorry PR, Cowley D, et al. Use of laser capture microdissection to detect integrated HIV-1 DNA in macrophages and astrocytes from autopsy brain tissues. J Neurovirol. 2006;12(2): 146-52. This paper is important because it showed, in a very prescient way and with great technical tour de force, that astrocytes in human brain specimens are very likely to harbor a substantial brain reservoir of "latent" HIV DNA. The push to cure HIV infection and eradicate latent HIV in the brain must confront the mechanism behind the critical findings that this paper reveals.

16. Everall IP, Heaton RK, Marcotte TD, et al. Cortical synaptic density is reduced in mild to moderate human immunodeficiency virus neurocognitive disorder. Brain Pathol. 1999;9:209-17.

17. Melrose RJ, Tinaz S, Castelo JM, et al. Compromised fronto-striatal functioning in HIV: an fMRI investigation of semantic event sequencing. Behav Brain Res. 2008;188(2):337-47.

18. Ernst T, Chang L, Jovicich J, et al. Abnormal brain activation on functional MRI in cognitively asymptomatic HIV patients. Neurology. 2002;59(9):1343-9. doi:10.1212/01.WNL. 0000031811.45569.B0.

19. Ances BM, Sisti D, Vaida F, et al. Resting cerebral blood flow: a potential biomarker of the effects of HIV in the brain. Neurology. 2009;73(9):702-8. This paper convincing shows that HIV-infected people with and without HAND exhibit abnormal blood flow patterns. The effect is not well understood and might be highly important biologically. Interesting brain regional variation occurs, which is potentially analogous to brain gene expression data that also varies by brain region cited in reference 21 . Combined with the avid interest in neurovascular biology in virally suppressed patients with HAND, the importance of this work is destined to grow with time

20. Gelman BB, Lisinicchia JG, Chen T, Johnson KM, Jennings K, Freeman Jr DH, et al. Prefrontal dopaminergic and enkephalinergic synaptic accommodation in HIV-associated neurocognitive disorders and encephalitis. J Neuroimmune Pharmacol. 2012;7(3):686700. doi:10.1007/s11481-012-9345-4. The study used over $\mathbf{5 0 0}$ autopsy brain specimens which permitted new insights regarding synaptic transmission in HAND. Those patients who downregulated their expression of dopamine 2 receptors in their prefrontal cortex performed better on neuropsychological testing than the patients who did not. That and other differences did not depend on the presence of encephalitis. The discussion makes a strong case that synaptic plasticity and accommodation occur in response to HIV infection

21.• Gelman BB, Chen T, Lisinicchia JG, Soukup VM, Carmical JR, et al. The national NeuroAIDS tissue consortium brain gene array: two types of HIV-associated neurocognitive impairment. PLoS One. 2012;7(9):e46178. doi:10.1371/journal.pone.0046178. This is the only brain gene array in patients with HAND that examines patients with and without encephalitis in multiple brain sectors. Using 72 arrays with over 56,000 probes each, a large consortium shows that two distinct neuropathological and brain transcriptional patterns occur in HAND. In virally suppressed patients without encephalitis, gene expression suggests that neurovascular biology is significantly involved. With encephalitis, inflammatory changes dominate and show a striking dependence on brain region, which strongly suggests that circuit-level dysfunction occurs in response to CNS inflammation.
22. Brew BJ, Crowe SM, Landay A, Cysique LA, Guillemin G. Neurodegeneration and ageing in the HAART era. $\mathrm{J}$ Neuroimmune Pharmacol. 2009;4:163-74. doi:10.1007/s11481008-9143-1. This is a highly useful review article that comprehensively covers an unwieldy and still-growing body of literature. One drawback to this otherwise excellent treatise is that excessive credence was given to studies with a large number of HIV-infected cases, while the adequacy of agematched control cases was ignored. But a more digestible handling of the topic than this has yet to appear.

23. Power C, Kong PA, Crawford TO, Wesselingh S, Glass JD, McArthur JC, et al. Cerebral white matter changes in acquired immunodeficiency syndrome dementia: alterations of the bloodbrain barrier. Ann Neurol. 1993;34(3):339-50.

24. Langford TD, Letendre SL, Marcotte TD, et al. Severe, demyelinating leukoencephalopathy in AIDS patients on antiretroviral therapy. AIDS. 2002;16(7):1019-29. doi:10.1097/00002030200205030-00008.

25. Jernigan TL, Archibald SL, Fennema-Notestine C, et al. Clinical factors related to brain structure in HIV: the CHARTER study. J Neurovirol. 2011;17(3):248-57. doi:10.1007/s13365-011-0032-7.

26. Hawkins BT, Davis TP. The blood-brain barrier/neurovascular unit in health and disease. Pharmacol Rev. 2005;57(2):173-85. doi:10. 1124/pr.57.2.4.

27. Hill J, Rom S, Ramirez SH, Persidsky Y. Emerging roles of pericytes in the regulation of the neurovascular unit in health and disease. J Neuroimmune Pharmacol. 2014;9(5):591-605. doi:10. 1007/s11481-014-9557-x.

28. Krause BW, Wijtenburg SA, Holcomb HH, et al. Anterior cingulate GABA levels predict whole-brain cerebral blood flow. Neurosci Lett. 2014;561:188-91.

29. Li JZ, Arnold KB, Lo J, et al. Differential levels of soluble inflammatory markers by human immunodeficiency virus controller status and demographics. Open Forum Infect Dis. 2014;2(1). doi:10. 1093/ofid/ofu117.

30. Massanella M, Ouchi D, Marfil S, et al. Different plasma markers of inflammation are influenced by immune recovery and cART composition or intensification in treated HIV-infected individuals. PLoS One. 2014;9(12):e114142. doi:10.1371/journal.pone.0114142.

31. Lyons JL, Uno H, Ancuta P, Kamat A, Moore DJ, Singer EJ, et al. Plasma sCD14 is a biomarker associated with impaired neurocognitive test performance in attention and learning domains in HIV infection. Acquir Immune Defic Syndr. 2011;57(5):371-9. doi:10.1097/QAI.0b013e3182237e54.

32. Ancuta P, Kamat A, Kunstman KJ, Kim EY, Autissier P, Wurcel A, et al. Microbial translocation is associated with increased monocyte activation and dementia in AIDS patients. PLoS One. 2008;3(6): e2516. doi:10.1371/journal.pone.0002516. This paper is important because it clearly illustrates how organs of the body other than the CNS play important roles in producing a systemic disease that encompasses HAND. The study was executed very elegantly with judicious use of resources. It stands as a prototypal example of how to effectively approach the clinicopathological correlation of HAND.

33. McArthur JC, Hoover DR, Bacellar H, et al. Dementia in AIDS patients incidence and risk factors. Neurology. 1993;43(11):224552. doi:10.1212/WNL.43.11.2245.

34. Kuller LH, Tracy R, Belloso W, et al. Inflammatory and coagulation biomarkers and mortality in patients with HIV infection. PLoS Med. 2008;5:e203. doi:10.1371/journal.pmed.0050203.

35. Wolf K, Tsakiris DA, Weber R, et al. Antiretroviral therapy reduces markers of endothelial and coagulation activation in patients infected with human immunodeficiency virus type 1 . J Infect Dis. 2002;185(4):456-62. doi:10.1086/338572.

36. Glass JD, Fedor H, Wesselingh SL, McArthur JC. Immunocytochemical quantitation of human immunodeficiency 
virus in the brain: correlations with dementia. Ann Neurol. 1995;38:755-62.

37. Siliciano RF, Greene WC. HIV latency. Cold Spring Harb Perspect Med. 2011;1(1):a007096. doi:10.1101/cshperspect.a007096.
38. Desplats P, Dumaop W, Smith D, et al. Molecular and pathologic insights from latent HIV-1 infection in the human brain. Neurology. 2013;80(15):1415-23. doi:10.1212/WNL.0b013e31828c2e9e. 